\title{
Fast solution techniques for corrosion and signatures modelling
}

\author{
A. J. Keddie ${ }^{1}$, M. D. Pocock ${ }^{1} \&$ V. G. DeGiorgi ${ }^{2}$ \\ ${ }^{1}$ Frazer-Nash Consultancy Ltd., Dorking, Surrey, UK \\ ${ }^{2}$ Naval Research Laboratory, Washington, DC, USA
}

\begin{abstract}
The boundary element method (BEM) involves field nodes interacting with each other. Solving the ensuing matrix equations often requires an iterative solver to be used at a cost that scales with the second power of the number of nodes per iteration. This limits the size of the problem that can be solved. The fast multipole method (FMM), introduces hubs to reduce the number of direct interactions between field nodes in the BEM. The cost of calculating the matrix-vector multiplication using the FMM scales linearly with problem size. This paper contains a brief mathematical description of the FMM for Laplace's equation in which a Taylor series expansion is used to model Green's function. The computational performance of the FMM applied to modelling an impressed current cathodic protection (ICCP) system of a naval vessel is then investigated and the results compared to those of a commercial BEM solver and experimental (physical scale model) results. For this relatively small example model, it is shown that the cost benefit of the FMM is eight times greater than that of the commercial solver. Greater savings will be obtained on larger models. The results confirm that larger, more detailed, corrosion problems can be solved faster using the FMM. It is also shown that the capabilities of the FMM offer the choice between reduced processing time and enhanced accuracy. This provides the user with the opportunity to sacrifice accuracy in order to run less computationally expensive problems, for example during parametric studies. Keywords: boundary element methods, fast multipole method, ICCP system.
\end{abstract}




\section{Introduction}

ICCP systems exploit the electrochemical nature of corrosion and the establishment of cathode-anode regions on the ship. Cathodes (exposed metal regions on the hull, rudder and propeller) are protected regions and anodes are either discrete components of sacrificial metal or electron source points positioned along the underwater hull at specific locations. For a ship at sea, the wetted surface area of the hull and the appendages such as bilge keel, rudder and propeller, are the cathodes, which require $\mathrm{CP}$.

A typical ICCP system consists of non-sacrificial noble anodes connected to power supplies, reference cells to monitor hull potential state and a controller to adjust the current output of the anodes. By ensuring that the on-board power supply provides controllable anodic current to inert anodes, the ICCP system inhibits corrosion of a material that would otherwise act as an anode by forcing it to behave cathodically. The ideal system is designed with anodes located so current is evenly distributed to ensure that a uniform voltage is maintained for all points on the underwater hull. In reality, cathodes created by paint damage, components made of non-similar materials, geometric features and openings in the hull result in a varied profile.

In the evaluation of material or system performance an appropriate material characterisation is necessary. In the case of electrochemical corrosion, the sensitivity of the electrical current-electrical potential relationship to environmental and electrolyte characteristics must be considered when defining an appropriate characterisation. The accuracy of corrosion prediction, whether based on analytical, experimental or computational analysis, will depend on how well the system defined in the analysis matches both the real structure and the environment surrounding the structure.

Polarisation, which for present purposes may be characterised as the relationship between electrical current and electrical potential, is an observed behaviour resulting from the combined effects of multiple oxidation and reduction reactions and is usually non-linear. This often complex behaviour between electrical current and potential severely complicates the process of corrosion prediction and sensitivity studies can be used as a method for determining the suitability of polarisation relationships.

\section{Ship geometry}

The geometry used in this study is the same as that used by DeGiorgi et al [1]. It is representative of navy ship hulls with one propeller and one rudder located along the port-starboard centerline. The ship is outfitted with a 2 zone ICCP system. Anode placement, power supply sizing, and reference cell locations are defined based on established design rules.

In [1], the authors used the boundary element code FNREMUS [2] to compare the performance of a 2 zone ICCP system for four zonal operational combinations. The focus of this study will be for the case of normal operating condition in which both forward and aft zones are fully functional. In [1], the 
authors compared computational results for this case with those obtained using physical scale modelling (PSM) for the same problem. PSM is a physics based experimental process used to design and evaluate ICCP systems. Details of this approach can be found in [3].

The authors have duplicated the geometry, ICCP system, material configuration, material properties and loading conditions defined in [1]. The ship hull used is representative of the type of ship that can be found in the US Navy but is not a duplicate of any active ship. The ICCP system is a conceptual system similar to that which may be found on US Navy ships. The ship hull boundary element mesh (Figure 1) is identical to that found in [1]. The commercial code PATRAN [4] was used to generate the ship hull mesh. Only the portion below the design waterline is included in the model. Results are calculated for the minimum damage $(3 \%$ bare material concentrated in the docking blocks and propeller) static flow condition. The intent is not to create a new analysis of the design but to compare solver CPU capabilities on a standard published problem. The ship model is submerged in a large container of seawater representing infinite expanse of open ocean. The electrolyte is modeled as scaled (1/96) seawater with a conductivity of $5.69 \times 10^{-2}$ Siemens $/ \mathrm{m}$. Material properties are identical to those used in [1]. Further details on material property definitions can be found in [5]. A reference cell set value of $-0.85 \mathrm{~V} \mathrm{Ag} / \mathrm{AgCl}$ electrode is used in the analysis.
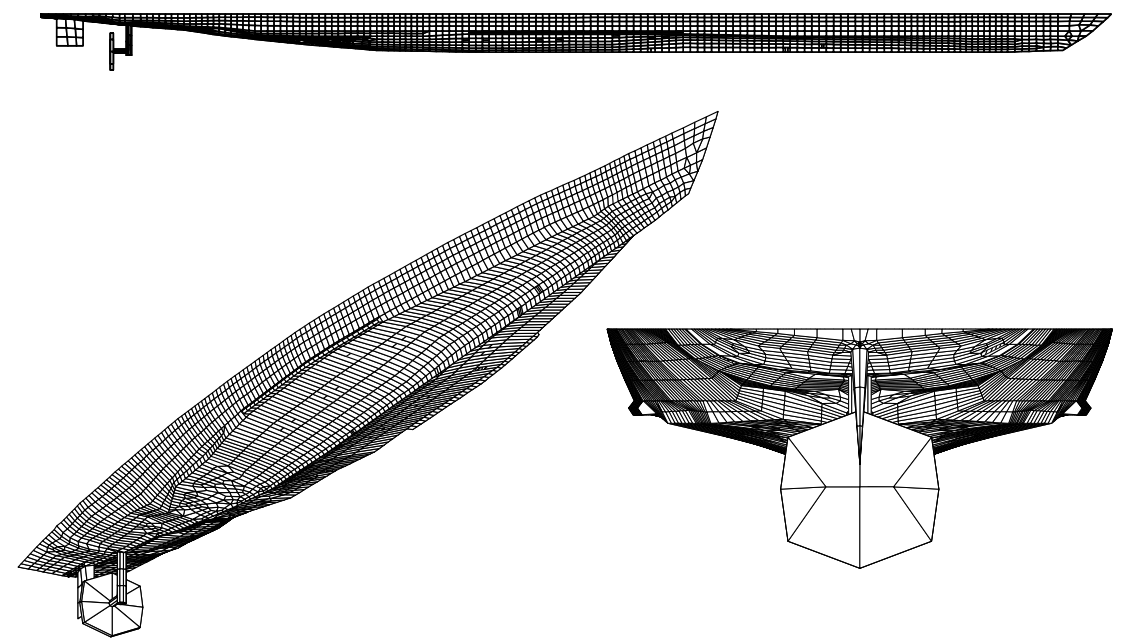

Figure 1: $\quad$ Boundary element mesh.

\section{ICCP controller model}

The solution algorithm is an iterative process in which the anode current for each iteration is determined from the difference between the reference cell's set 
potential and achieved potential at the previous iteration. The relationship between potential and current can be a simple linear gain, a ProportionalIntegral-Derivative (PID) type control or a more complex algorithm, and can take account of maximum supply currents or other physical constraints of the ICCP system. At each iteration the currents at all anodes are adjusted to minimize the difference between set and achieved potential at each reference cell. This involves employing another iterative process around the (usually) nonlinear material polarisation relationship.

The surface potential on a vessel has traditionally been calculated at each iteration by employing the boundary element method (BEM) to numerically solve Laplace's equation. Boundary element techniques require solving a system of equations for which the computational cost scales with the second power of the problem size. This becomes prohibitively large as the number of nodes for a particular problem increases. As will be discussed in the following section, the fast multipole method (FMM) reduces this computational and storage cost by approximating the integrals within the BEM.

Table 1: $\quad$ ICCP system details-scale geometry dimensions, [1].

\begin{tabular}{|l|c|c|c|c|}
\hline & Frame & $\begin{array}{c}\text { Below } \\
\text { Waterline }(\mathrm{cm})\end{array}$ & $\begin{array}{c}\text { Off Centerline } \\
(\mathrm{cm})\end{array}$ & Symmetry \\
\hline $\begin{array}{l}\text { Forward Zone } \\
\text { Anodes }\end{array}$ & 49.9 & 2.3 & 1.8 & $\begin{array}{c}\text { Port \& } \\
\text { Starboard }\end{array}$ \\
\cline { 2 - 5 } & 189.1 & 2.8 & 4.6 & $\begin{array}{c}\text { Port \& } \\
\text { Starboard }\end{array}$ \\
\hline $\begin{array}{l}\text { Forward Zone } \\
\text { Reference Cells }\end{array}$ & 103.7 & 3.3 & 1.0 & $\begin{array}{c}\text { Port \& } \\
\text { Starboard }\end{array}$ \\
\hline \multirow{2}{*}{$\begin{array}{l}\text { Aft Zone } \\
\text { Anodes Zon \& }\end{array}$} & 279.4 & 2.8 & 4.3 & $\begin{array}{c}\text { Port \& } \\
\text { Starboard }\end{array}$ \\
\cline { 2 - 5 } & 368.8 & 0.7 & 3.8 & Port \\
\hline $\begin{array}{l}\text { Aft Zone } \\
\text { Reference Cell }\end{array}$ & 397.0 & 0.5 & 3.8 & Starboard \\
\hline
\end{tabular}

\section{Numerical methods for solving Laplace's equation}

\subsection{Boundary element modelling and cost scalings}

Within each iteration of the FNREMUS code it is necessary to solve Laplace's equation. Laplace's equation, which models the electric field, is expressed as

$$
\nabla^{2} \phi(\mathbf{y})=0
$$

for some location $\mathbf{y}$.

Green's function for Laplace's equation is:

$$
G(\mathbf{x}, \mathbf{y})=\frac{1}{4 \pi|\mathbf{x}-\mathbf{y}|}
$$


The Boundary Integral form for Laplace's equation can be expressed as:

$$
\frac{1}{2} \phi(\mathbf{x})=\int_{\partial \Omega}\left(G(\mathbf{x}, \mathbf{y}) \frac{\partial \phi}{\partial n^{\prime}}-\phi \frac{\partial}{\partial n^{\prime}}(G(\mathbf{x}, \mathbf{y}))\right) d s^{\prime}
$$

where $d s^{\prime}$ indicates that we are integrating over the surface with respect to $\mathbf{y}$.

Discretising (3) and evaluating the integrals using Gaussian quadrature, gives the following system of equations:

$$
2 \pi \phi_{i}+\sum_{j=1}^{N} a_{i j} \phi_{j}-\sum_{j=1}^{N} b_{i j}\left(\frac{\partial \phi}{\partial n^{\prime}}\right)_{j}=0
$$

where, $N$ represents the number of nodes in the problem after discretising. In matrix form this gives:

$$
\left[\begin{array}{ll}
A & \\
&
\end{array}\right]\left[\begin{array}{ll}
\phi & B
\end{array}\right]\left[\begin{array}{l}
\phi^{\prime} \\
\end{array}\right]
$$

where $\phi^{\prime}=\partial \phi / \partial n^{\prime}$.

The cost of storing and evaluating (5), using an indirect solver, scales with the second power of the size of the problem. A problem that doubles in size will therefore approximately quadruple in memory requirement and computational time. This becomes prohibitively large as the size of $N$ approaches $10^{5}$.

\subsection{Fast multipole method and cost scalings}

One of the methods that has promised the most in recent years, with respect to reducing the operation count and storage requirements of the BEM is the fast multipole method. The FMM was initially introduced by Rokhlin [6] as a fast solution method for the two-dimensional Laplace equation. The FMM can be thought of as a method for separating the kernel of the BEM into a product of functions of its dependant variables, i.e. if we can write

$$
G(\mathbf{x}, \mathbf{y})=g_{1}(\mathbf{x}) g_{2}(\mathbf{y})
$$

In this case we may re-express (3), after moving the $g_{1}$ term outside of the integral, as

$$
\frac{1}{2} \phi(\mathbf{x})=g_{1}(\mathbf{x}) \int_{\partial \Omega}\left(g_{2}(\mathbf{y}) \frac{\partial \phi}{\partial n^{\prime}}-\phi \frac{\partial}{\partial n^{\prime}}\left(g_{2}(\mathbf{y})\right)\right) d s^{\prime}
$$

We see that in (7), $g_{1}$ and $g_{2}$ take on $N$ different values. The calculation of the integral will therefore require just order $N$ operations, and the evaluation of $g_{1}$ at all locations $\mathbf{x}$, a further order $N$ operations.

Most kernels of interest cannot be expressed directly in such a simple form as (7) and the FMM can be thought of as a way of achieving such a decomposition, by in part, expanding the kernel as an infinite series. Expanding the function $F(\mathbf{x}, \mathbf{y})$, around a reference point, $\mathbf{z}$, using a Taylor series expansion gives:

$$
F(\mathbf{x}, \mathbf{y})=\sum_{\beta=0}^{\infty} \frac{1}{\beta !}((\mathbf{z}-\mathbf{y}) \cdot \nabla)^{\beta} F(\mathbf{x}, \mathbf{z})
$$

which, for simplicity, can be expressed as a truncated series of products of functions that depend on $\mathbf{x}, \mathbf{y}$ and $\mathbf{z}$ : 


$$
F(\mathbf{x}, \mathbf{y}) \approx \sum_{\beta=0}^{p} f_{1}^{(\beta)}(\mathbf{z}, \mathbf{y}) f_{2}^{(\beta)}(\mathbf{x}, \mathbf{z})
$$

Expressing Green's function in the form of (9) and substituting into (3) gives

$$
\frac{1}{2} \phi(\mathbf{x})=\sum_{\beta=0}^{p} g_{1}^{(\beta)}(\mathbf{x}, \mathbf{z}) \int_{\partial \Omega}\left(g_{2}^{(\beta)}(\mathbf{z}, \mathbf{y}) \frac{\partial \phi}{\partial n^{\prime}}-\phi \frac{\partial}{\partial n^{\prime}}\left(g_{2}^{(\beta)}(\mathbf{z}, \mathbf{y})\right)\right) d s^{\prime}
$$

As with many series expansions, the rate of convergence is of utmost importance. Such expansions converge efficiently only for small ranges of the dependant variables. Indeed for the Taylor series expansion, and hence (10), to converge, the condition that $|\mathbf{z}-\mathbf{y}|<|\mathbf{x}-\mathbf{z}|$ must be satisfied. As such, it is not possible to evaluate (10), for all locations $\mathbf{x}$ and $\mathbf{y}$, by choosing a single value of $\mathbf{z}$.

To overcome this limitation, we introduce a hierarchical decomposition of the spatial domain to carry out grouping of nodes. This is achieved by decomposing the domain into a finite number of groups, $\partial \Omega_{l}$, each containing a centre point $\mathbf{z}_{l}$, such that (10) can be expressed as

$$
\frac{1}{2} \phi(\mathbf{x})=\sum_{\beta=0}^{p} \sum_{l} g_{1}^{(\beta)}\left(\mathbf{x}, \mathbf{z}_{l}\right) \int_{\partial \Omega_{l}}\left(g_{2}^{(\beta)}\left(\mathbf{z}_{l}, \mathbf{y}\right) \frac{\partial \phi}{\partial n^{\prime}}-\phi \frac{\partial}{\partial n^{\prime}}\left(g_{2}^{(\beta)}\left(\mathbf{z}_{l}, \mathbf{y}\right)\right)\right) d s^{\prime}
$$

Evaluation of (11) can be performed for nearly every $\mathbf{x}$ and $\mathbf{y}$ location. This is achieved by only allowing collocation nodes, $\mathbf{x}$, to interact with relatively distant groups of integration nodes, $\mathbf{y}$, with centre $\mathbf{z}_{l}$. The FMM goes on to decrease the computational cost by performing additional expansions. For example, the functions $g_{1}$ and $g_{2}$, which, like (9) depend on two vectors, can also be expressed as Taylor series expansions. By doing so, the number of direct interactions between nodes is decreased. This is illustrated in Figure 2 for the BEM and FMM.
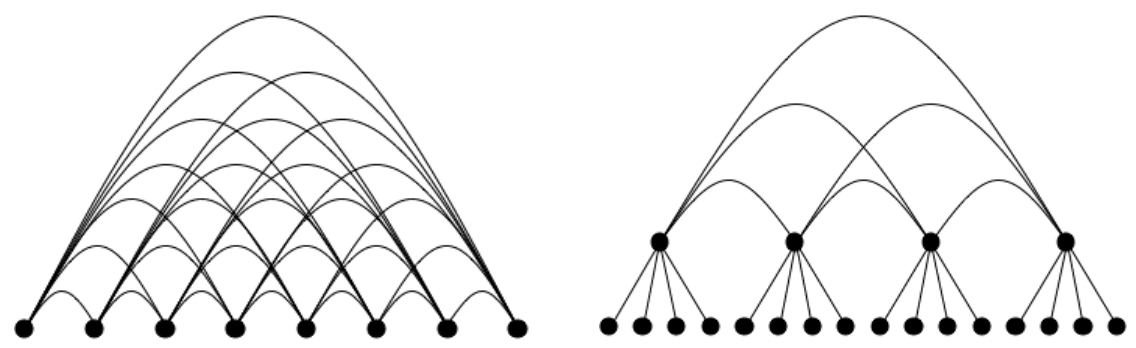

Figure 2: Diagram showing the interaction of nodes in the BEM (left) and FMM (right). Hubs are established in the FMM to reduce the number of nodal links and hence the computational cost.

While performing additional expansions produces a complex grouping structure, doing so will result in the computational and storage cost of the FMM to scale approximately with the size of the problem, $N$. Clearly, the reduction in cost, will allow larger problems to be solved faster. Indeed, even for the modestly sized surface ship mesh, described in Section 2, it would be expected 
that significant savings in CPU time would be achieved using the FMM. This offers the time-constrained user the ability to perform more detailed sensitivity studies than would otherwise be possible. Additionally, the CPU-time required to solve a problem can be further decreased by reducing the number of expansion terms, $p$, used. This is likely to cause a slight reduction in the accuracy of the overall solution since the relevant function in (9) will be approximated using fewer terms. However, this is a useful tool in the initial stages of sensitivity analyses and allows the user the chance to perform preliminary studies in relatively quick time before performing more accurate and detailed analyses.

\section{Results}

In this section computational results using two Laplace solvers within FNREMUS will be studied. These are:

- BEASY-Thermal, a commercial boundary element solver; and

- the FMM code as described in Section 4.

These will henceforth be referred to as FNREMUS-BEASY and FNREMUS-FMM, respectively. Experimental results, obtained using physical scale modelling (PSM), will also be used to validate the computational results. Results calculated using both PSM and FNREMUS-BEASY were calculated as part of the paper by DeGiorgi et al [1].

In order to compare its performance and accuracy, the results calculated using FNREMUS-FMM were obtained using different numbers of expansion terms. While increasing the number of terms in the Taylor series expansion will generally increase the accuracy of the solution, the benefits of doing so must be offset against the additional computational cost of calculating the extra expansion terms.

Key results obtained using PSM and computational methods are shown in Table 2. As discussed by DeGiorgi et al [1] variations can be seen as indications of the non-uniqueness of the multiple anode-multiple cathode-variable input current problem. This issue is not discussed here since the objective of this work is the comparison of solver performance. Of importance here is the comparison between the two calculated results. In both FNREMUS solvers, the current from the anodes will equal the sum of the current from the propellers and docking block. It can be seen from FNREMUS-BEASY results that the total anodic and cathodic currents are equal to $1.973 \mathrm{~mA}$. While the total anodic current shows good agreement with the PSM results, the individual (fore and aft) currents show differences. This is the same pattern as seen in [1]. The FNREMUS-FMM results in Table 2 show that there is a total anodic and cathodic current of between $2.011 \mathrm{~mA}$ and $2.009 \mathrm{~mA}$ depending on the number of expansion terms used. The difference between FNREMUS-FMM and PSM anode currents is greater than the differences calculated for FNREMUS-BEASY and PSM. However, there is a smaller difference between the total cathodic currents for FNREMUS-FMM and PSM. Indeed, the FNREMUS-FMM values of anodic/cathodic currents are closer to the average of the PSM anodic/cathodic currents than FNREMUS-BEASY. As with the FNREMUS-BEASY case, the 
fore and aft currents show differences between PSM and FNREMUS-FMM. This could again be attributed to multiple combinations of zonal input values that result in similar overall system performance parameters.

Table 2: $\quad$ Corrosion results from ship hull calculated using Physical Scale Modelling (PSM), FNREMUS-BEASY and FNREMUS-FMM.

\begin{tabular}{|l|c|c|c|c|c|c|}
\cline { 4 - 7 } \multicolumn{1}{c|}{} & PSM & FNREMUS- & \multicolumn{4}{c|}{ FNREMUS-FMM } \\
\cline { 4 - 7 } \multicolumn{1}{c|}{} & & BEASY & $\mathrm{p}=2$ & $\mathrm{p}=3$ & $\mathrm{p}=4$ & $\mathrm{p}=5$ \\
\hline Fore anodes $(\mathrm{mA})$ & -1.21 & -0.161 & -0.143 & -0.183 & -0.182 & -0.204 \\
\hline Aft anodes $(\mathrm{mA})$ & -0.76 & -1.812 & -1.868 & -1.828 & -1.828 & -1.805 \\
\hline Total & -1.97 & -1.973 & -2.011 & -2.011 & -2.010 & -2.009 \\
\hline Docking blocks $(\mathrm{mA})$ & 0.89 & 0.889 & 0.915 & 0.915 & 0.914 & 0.913 \\
\hline Propeller (mA) & 1.15 & 1.084 & 1.097 & 1.096 & 1.096 & 1.096 \\
\hline Total & 2.04 & 1.973 & 2.011 & 2.011 & 2.010 & 2.009 \\
\hline $\begin{array}{l}\text { Forward reference } \\
\text { electrode }(\mathrm{mV})\end{array}$ & -850 & -851 & -851 & -851 & -851 & -851 \\
\hline $\begin{array}{l}\text { Aft reference } \\
\text { electrode }(\mathrm{mV})\end{array}$ & -850 & -850 & -849 & -849 & -849 & -849 \\
\hline
\end{tabular}

Figure 3 shows the CPU times required to run the model using FNREMUSBEASY and FNREMUS-FMM with increasing expansion terms. The total CPU time taken to run FNREMUS-BEASY is approximately 24,000 seconds. This is at least twice as long at it takes to run FNREMUS-FMM for any of the cases shown. The time taken to solve the problem using FNREMUS-FMM with two expansion terms is approximately 3,000 seconds or eight times quicker than FNREMUS-BEASY. As larger problems are solved, the difference in time taken to solve them using both FNREMUS solvers will be exacerbated due to the cost scalings of each method.

It can be seen that the currents in Table 2 don't appear to converge towards a single value as the number of expansion terms increases. This is most likely a result of the FNREMUS ICCP algorithm which employs an iterative solution technique around the non-linear material polarisation relationship. The currents shown in Table 2 are the first iterative values within the specified tolerance.

\section{Summary}

In this work the fast multipole method has been successfully applied to the computational modelling of electrochemical corrosion on a surface ship. The ship chosen is one similar to US Navy ships with a system similar to that which can be found on these ships. The problem chosen is representative of the type of design problem which may be encountered and does have a basis in reality. Cost savings estimated in this work would therefore translate to real cost savings. The CPU-time required to solve this problem was more than eight times faster than a commercial boundary element when solved using two expansion terms 
and twice as fast when using five terms. Furthermore, the results obtained using the FMM corrosion solver demonstrate the same type of agreement and variation with PSM as shown earlier with conventional BEM solvers [1]. This indicates that there are no additional errors introduced into the modelling process by the use of the fast multipole method.

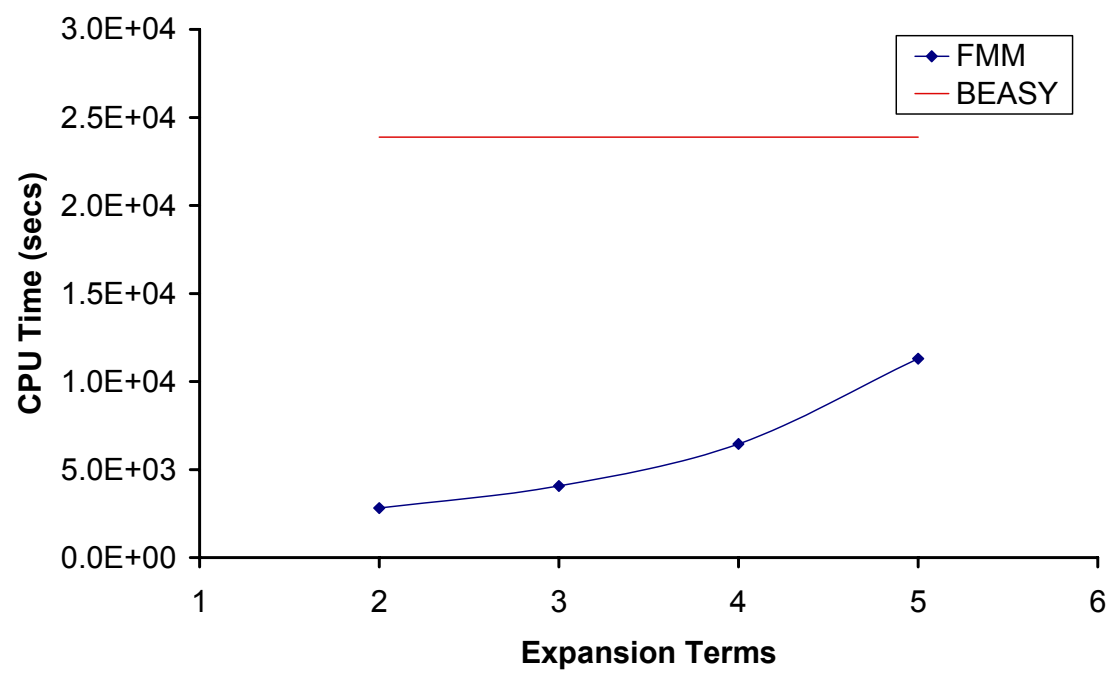

Figure 3: CPU time required to run FNREMUS using different Laplace solvers on a Frigate model with ICCP system. FNREMUS-BEASY was only run once but has been shown here for comparison with FNREMUS-FMM.

Based on the comparison of runtimes generated by analysis of a realistic ship problem, it has been determined that incorporation of the fast multipole method into $\mathrm{BE}$ code would greatly enhance the ability to use BEM for large problem solutions. This could readily lead to more use by those interested in the solution of electric fields on- and off-board of large complex structures such as modern military ships.

\section{References}

[1] DeGiorgi, V.G., Pocock, M.D., Wimmer, S.A., \& Hogan, E.A., "Zonal ICCP System Control Interactions," Simulation of Electrochemical Processes, 15-24, 2005.

[2] Frazer-Nash Consultancy, FNREMUS Detailed Modeller User Guide, FNC 5421/21133R, Issue 1, 2000.

[3] DeGiorgi, V.G., Hogan, E., Lucas, K.E. \& Wimmer, S.A., "Shipboard Impressed Current Cathodic Protection System," Chapter 2, Modeling of Cathodic Protection Systems, WIT Press, 13-44 2006. 
234 Simulation of Electrochemical Processes II

[4] MSC Software Corp., MSC Patran 2001 User's Manual, 2001.

[5] DeGiorgi, V.G., "Evaluation of Perfect Paint Assumptions in Modeling of Cathodic Protection Systems," Engineering Analysis with Boundary Elements, 26/5, 435-445, 2002.

[6] Rokhlin, V., "Rapid Solution of Integral Equations of Classical Potential Theory," Journal of Computational Physics, 1985. 60: 187-207. 